\title{
Flexibility Characterization of a Residential Neighbourhood with Water-to-water Heat Pumps Using Model Predictive Control
}

\author{
Frédéric Amblard ${ }^{1,2}$, Ramanunni P. Menon ${ }^{1}$, Jessen Page ${ }^{1}$ \\ ${ }^{1}$ HES-SO Valais-Wallis, Sion, Switzerland \\ ${ }^{2}$ Ecole Polytechnique Fédérale de Lausanne (EPFL), Lausanne, Switzerland
}

\begin{abstract}
Demand Response is an attractive option to solve the problem of balancing supply and demand while providing flexibility to the grid. Buildings have been identified as highly valuable flexibility resources due to the increase of thermoelectric devices such as heat pumps for space heating and Domestic Hot Water (DHW). However, current methods for estimating the flexibility potential for residential buildings tend to rely on simplified models and often overlook the limitation of on-site control systems, especially for complex thermoelectric devices such as heat pumps. This contribution aims at accounting for this in assessing the flexibility offered by heat pumps for several market-based indicators using a simulation model and Model Predictive Control (MPC). Simulations are carried out to evaluate the proposed formulations and metrics, especially in relation to maximum flexibility potential fluctuations over a day or between seasons. Our results show that flexibility varies throughout the year, with maximum values reached at midseason. Our results also emphasize the importance of the choice of metrics to assess flexibility.
\end{abstract}

\section{Introduction}

The increase of stochastic and decentralized renewable energy sources (e.g. photovoltaics, wind turbines) makes balancing short/medium-term electric supply and demand difficult. This means that more flexibility will be required from power systems. The traditional approach of power suppliers to tackle this problem is top-down: the power generation is controlled to fulfill the energy demand curves. However, an attractive alternative to adjust demand to the available production is the so-called Demand Response (DR). DR, defined by the Strategic Energy Technologies Information System (SETIS) of the European Commission, is an "intentional modification of normal consumption patterns by end-use customers in response to incentives from grid operators".

Supply of energy services in urban areas corresponds to more than $60 \%$ of global energy consumption, according to Van der Hoeven (2012), and most of this energy is related to buildings. The two main consumption sources are space heating and Domestic Hot Water (DHW) accounting together for around $80 \%$ of the total energy consumption of buildings. Shifting the energy consumption for heating and DHW in buildings is thus a lucrative way of applying DR. The capacity of residential buildings to be able to intentionally modify their consumption patterns is often referred as their "flexibility". This potential is affected by several human, technical and external factors: (1) the inhabitant's behaviour and their comfort requirements, (2) the building characteristics such as the thermal capacity and the insulation, (3) the heat distribution system including thermoelectric devices, storage tanks and heat exchangers, (4) their control system and the degree of monitoring, and (5) the weather conditions.

An attractive method to perform DR is model predictive control (MPC). It can maximize the flexibility potential while respecting system constraints and user comfort. In this contribution, we evaluate the flexibility offered by heat pumps in residential buildings while using MPC to perform DR. We compare our estimates of flexibility to a more standard method to perform DR actions called Rule Based Control (RBC). Since the other DR solutions available on the market are mostly limited to electricity (PV, electric batteries, electric heaters) we concentrate our efforts to the same.

We characterize flexibility at subhourly time steps for residential buildings with a focus on the effect of two control methods. We investigate the choice of flexibility metrics and discuss the reference used. The potential is assessed with the example of a simulated cluster of buildings, which is supplied by water-towater heat pumps, during typical days.

After a review of related work in flexibility characterization and similarity measures for time series, the second section introduces the framework used to investigate the flexibility potential of a residential neighbourhood. Several flexibility indicators and the methods to compute them are presented along with the evaluated control algorithms. The use of typical days 
is elaborated on. Results and a discussion of the indicators, the methods and the control algorithms presented is found in the third section.

\section{Related work}

\section{Flexibility definition}

The term "Energy Flexibility" is used in many different domains related to electric power systems but is understood differently by physicists, thermodynamicists or control engineers, which leads to almost as many definitions as papers published. It still lacks a universally accepted definition. For this reason, Reynders et al. (2018) review and evaluate existing definitions and quantification methodologies applied to flexible energy in buildings. They found that for systems with multiple time constants, such as buildings, it is more appropriate to quantify flexibility by analyzing flexibility events at specific time points rather than relying on cumulative energy profiles. They identified several focus points but they did not provide an overall definition for flexibility.

\section{Flexibility potential estimation}

There are even more indicators and quantification methodologies than there are definitions for energy flexibility. Indeed, it can hardly be described by a single indicator (Stinner et al., 2016). In this paper, the authors develop a method that integrates the particular aspects of both the electrical grid and the building energy system. However, their work relies on a simplified model that does not account for the control aspects nor variation in efficiency, which is especially important when analyzing complex thermoelectric devices such as heat pumps.

The literature presents several recurrent concepts that are derived to compute the different indicators. The metrics often include the concept of time, power, energy and cost which can be connected to create more specific indicators. Zhang et al. (2019) present different DR applications with a focus on defining many indicators specific to each actor of the energy sector. They rely on a detailed structure of a residential energy system and two algorithms enabling to test "partial" and "full" DR call. Compared to most studies, they also consider DHW and account for rebound effect. However their flexibility estimation is based on simulated scenarios rather than using optimization control algorithms.

Junker et al. (2018) propose to define a "flexibility function", which enables the description of energy flexible transients without relying on a baseload estimation. The system is, however, assumed time invariant and linear. They further make the assumption that the system providing flexibility is smart and able to respond to an external penalty signal, which is not true yet in most buildings.

Most of the flexibility potential estimations for residential buildings tend to rely on simplified models.
Only a few papers, describe results obtained from pilot, such as the one presented by Dhulst et al. (2015). Experiments show that the flexibility potential throughout the day is "highly asymmetric".

In order to cover the gaps in literature mentioned above, we developed a simulation and optimization framework accounting for the effect of local control constraints. We account for the fact that the system can fail to comply with external penalty signals. Our framework allows us to test several DR configurations, especially in relation to maximum flexibility potential fluctuations over a day or between seasons. In this paper, we define flexibility as "any feasible change in power (increase or decrease) for a particular system, over a period of time, based on signals from the market/grid". Here, power refers only to electricity and not thermal power.

\section{Methods}

In this section, the flexibility metrics used in this case are introduced. Two control algorithms to provide flexibility are presented: RBC and MPC. They are used to dynamically change systems set-points to react to Demand Response calls. Then, we discuss a clustering method to reduce simulation time while ensuring a good assessment of flexibility potential over a year. Finally, the general framework is presented with focus on the parameters used to evaluate the flexibility potential of buildings. Note that the framework presented here is specific for residential buildings heated by Water-to-Water Heat Pumps (WWHP), but can be generalized for other systems and technologies.

\section{Indicators and metrics}

As a reminder, we define flexibility as any feasible change in power (increase or decrease) for a particular system, over a period of time, based on signals from the market/grid. Here, power refers only to electricity and not thermal power. We also consider only the controllable loads of buildings, such as heat pumps and electrical heaters.

The first indicator accounts for the maximum power deviation of a cluster. Given the index of the heat pump $n$ with $1 \leq n \leq N$, the Flexhour index $t_{0}$ $0 \leq t_{0} \leq 23$, corresponding to the hour of the call, the call duration $\left(d_{D R}\right)$ and the time index $t$ with $t_{0} \leq t \leq\left(t_{0}+d_{D R}\right)$, the amount of aggregated power consumption load $P$ that can be increased $(U P)$ to a maximum or reduced $(D N)$ to a minimum operation level is computed with the following two approaches. The first one computes the maximum power difference relative to the current power level at $t_{0}$ as in equations (1) and (2), while the second, equation (3), is based on the difference with the baseload power $P^{B L}$. The terms $U P$ and $D N$ have to be differentiated from the existing upward and downward reserve services provided by conventional generators. 
In this work, "Upward" (UP) refers to an increase in power consumption, which, looking at the market side, is "equivalent" to a decrease in power production (downward reserve).

$$
\begin{aligned}
& F_{1 t_{0}}^{U P}=\sum_{n=1}^{N} \frac{\max \left(P_{n, t}-P_{n, t_{0}}\right)}{P_{n}^{55^{\circ} \mathrm{C}}} \\
& F_{1_{t_{0}}}^{D N}=\sum_{n=1}^{N} \frac{\max \left(P_{n, t_{0}}-P_{n, t}\right)}{P_{n}^{55^{\circ} \mathrm{C}}}
\end{aligned}
$$

with $P_{n, t_{0}}$ the current power level and $P_{n}^{55^{\circ} \mathrm{C}}$ the power level of the heat pump when operating at a departure temperature of the condenser at $55^{\circ} \mathrm{C}$.

$$
F_{2 t_{0}}^{U P}=\sum_{n=1}^{N} \frac{\max \left(P_{n, t}-P_{n, t}^{B L}\right)}{P_{n}^{55^{\circ} C}}
$$

Both maximum deviation indicators $F$ are normalized by the power consumed in DHW mode with an operating temperature of $55^{\circ} \mathrm{C}$.

The second indicator describes the energy deviation in kWh (FlexEnergy) for a cluster of buildings for a specific duration call $d_{D R}$. Similar to the first set of indicators, these indicators are computed with two methods. The first one, equation (4), assumes that the current energy consumption at $t_{0}$ is maintained over the entire call duration. The second, equation (5), is based on the difference with the baseload energy consumption. Here, $P$ values are discrete with a sampling time $t s$ in seconds.

$$
\begin{aligned}
& E_{1 t_{0}}^{U P}=\frac{t s}{3.6 e^{6}} \sum_{n=1}^{N} \sum_{t=t_{0}}^{t_{0}+d_{D R}}\left(P_{n, t}-P_{n, t_{0}}\right) \\
& E_{2 t_{0}}^{U P}=\frac{t s}{3.6 e^{6}} \sum_{n=1}^{N} \sum_{t=t_{0}}^{t_{0}+d_{D R}}\left(P_{n, t}-P_{n, t}^{B L}\right)
\end{aligned}
$$

The third indicator tries to assess the rebound effect. It is computed as the energy deviation after the end of a DR call with the baseload as reference. For computational reasons, the rebound is computed for a period $d_{\text {rebound }}$ of four hours following the DR call.

$$
R_{t_{0}}=\frac{t s}{3.6 e^{6}} \sum_{n=1}^{N} \sum_{t=t_{0}+d_{D R}}^{t_{0}+d_{\text {rebound }}}\left(P_{n, t}-P_{n, t}^{B L}\right)
$$

The time taken to satisfy and maintain the change in power is an important metric of the quality of the flexibility supplied by the system. The changes in consumption patterns also have to respect the technical constraints of the systems and not compromise the comfort of users. This implies the need for a control methodology that can predict power curves and adapt over time, and makes MPC a good candidate for this work.

\section{Control}

Many studies discard the control aspect based on the assumption that "the system providing the flexibility is smart in a manner that it is able to respond to an external penalty signal" (Junker et al., 2018). On the contrary, we consider the local control aspect, as most systems cannot be forced but need to be encouraged to react to penalty signals. We assume that the heat pump power is modulated only by the different operation temperatures and between two states (on/off), as most of installed devices do. Three main manipulated variables are identified and used to control the heat pumps.

1. The indoor temperature set-point $T^{i n_{s}}$ used to compute the supply temperature $T^{\text {sup }_{s}}$ via the equation of the heat curve.

2. The DHW temperature set-point $T^{D H W_{s}}$ at which the HP will start heating the DHW storage tank.

3. The state of the HP $S^{H P}$ is either off, the HP is shut down, or on the HP can be, but is not necessarily, switched on.

The default control for each building is simulated by a heat curve, equation (7), and several dead-band controllers for the storage tank, with specific cut-in and cut-out temperature.

$$
T^{\text {sup }_{s}}=\operatorname{Slope}\left(T^{i n_{s}}-\overline{T^{e x t}}\right)+T^{i n_{s}}+\text { Level },
$$

where $\overline{T^{e x t}}$ is the average external temperature over a period of time.

At each specific DR time call, flexibility is evaluated for two different control approaches: RBC and MPC. In the case of RBC, the default control is run until a DR call to provide upward or downward flexibility is received. From this point onward and for the whole duration of the call $d_{D R}$, set-point values are put to their maximum/minimum values respectively. After the call, the set-points are set back to default values. The MPC maximizes flexibility potential while respecting system constraints and user comfort. The MPC implementation is deterministic in nature and uses weather data collected from Meteonorm (Remund and Kunz, 1995). The time horizon of the optimisation $h_{p}$ is equal to 24 hours, with a timestep $\Delta t$ of 15 minutes. The MPC cost function in equation (8), is a representation of the operational costs including the electricity price, the discomfort and the penalties for changing the behaviour from the base load. The optimization problem can be formulated as:

$$
\begin{array}{r}
\min \sum_{n=1}^{N}\left[\sum_{t=t_{0}}^{h_{p}}\left[c^{e l} P_{n, t}+c^{t c} \Delta T_{n, t}^{t c}+c^{a c t} \Delta u_{n, t}\right]\right. \\
\left.+\sum_{k=t_{0}}^{t_{0}+d_{D R}} c^{p e n} \Delta P_{n, k}\right]
\end{array}
$$




$$
\begin{array}{lr}
\text { Subject to: } & x_{t+1}=A x_{t}+B^{u} u_{t}+B^{w} w_{t} \\
\forall n, k: & \Delta P_{n, k} \geq P_{n}^{\max }-P_{n, k} \\
\forall n, t: & P_{n, t} \geq 0 \\
\forall n, t: & \Delta T_{n, t}^{t c} \geq T_{n, t}^{\min }-T_{n, t} \\
\forall n, t: & \Delta T_{n, t}^{t c} \geq T_{n, t}-T_{n, t}^{\max } \\
\forall n, t: & \Delta T_{n, t}^{t c} \geq 0
\end{array}
$$

In this formulation, the electricity price $c^{e l}$ is considered constant over the optimisation period. Equation (8b) quantifies the power deviation during the whole duration of the call $d_{D R}$ compared to the maximum power limit that is wished to be reached when responding to a upward flexibility request. The associated penalty cost, $c^{p e n}>>c^{e l}$, drives the objective function towards maximizing the power consumption even for rather short duration $\left(d_{D R}=15 \mathrm{~min}\right)$ compared to the prediction horizon $\left(h_{p}=24 h\right)$. The cost function also includes the level of discomfort $\Delta T_{n, t}^{t c}$, described in equation (8d-8f), with an associated penalty cost. It ensures that the state variables describing the air and mass temperature of the building as well as the DHW storage and buffer tanks stay within the comfort limits given by $T_{n, t}^{\min }$ and $T_{n, t}^{\max }$. Finally, $\Delta u_{n, t}$ quantifies the change of the manipulated variables. In order to penalize frequent control actuation that could alter the system performances, a cost $c^{a c t}$ is associated with $\Delta u_{n, t}$. The higher the cost, the less actuations are permitted.

The system models in equation (8a) are simplified representations of the devices used in the pilot site. A second-order state space model is used to represent the thermal inertia within the building. Contrary to first-order models, it is able to account for the two main time constants of the building: the fast dynamics of the air, used for assessing the comfort, and the slow dynamics of the internal mass, used for assessing the storage capacity. First-order equations are used for DHW storage tanks and space heating buffers.

The heat pumps are modelled by adapting the equations used by Schütz et al. (2015) to accommodate for the additional constraints imposed by the local controllers of the heat pumps. The new formulation in equations (9a-11b), allows to optimize a heat pump providing both space heating and DHW, contrary to the original formulation where only one of the two options is possible. When the heat pump produces DHW, the space heating distribution system is still active, supplied only by the energy stored in the heat buffer tank.

$$
\begin{array}{lr}
\forall n, t: & S_{n, t}^{s h}+S_{n, t}^{d h w} \leq 1 \\
\forall n, t: & S_{n, t}^{H P} \geq S_{n, t}^{s h} \\
\forall n, t: & S_{n, t}^{s h}+S_{n, t}^{d h w}=w 1_{n, t}+w 2_{n, t}+w 3_{n, t} \\
\forall n, t: & \operatorname{SOS} 2\left(w 1_{n, t}, w 2_{n, t}, w 3_{n, t}\right),
\end{array}
$$

where $S_{n, t}^{s h}, S_{n, t}^{d h w}$ and $S_{n, t}^{H P}$ are binary variables and $w 1_{n, t}, w 2_{n, t}$ and $w 3_{n, t}$ are weights [0,1].

$$
\begin{aligned}
& \forall n, t: Q_{n, t}^{h p}=Q_{35} w 1_{n, t}+Q_{45} w 2_{n, t}+Q_{60} w 3_{n, t} \\
& \forall n, t: P_{n, t}=P_{35} w 1_{n, t}+P_{45} w 2_{n, t}+P_{60} w 3_{n, t} \\
& \forall n, t: S_{n, t}^{s h}+S_{n, t}^{d h w} \leq 1 \\
& \forall n, t: S_{n, t}^{H P} \geq S_{n, t}^{s h} \\
& \forall n, t: Q_{n, t}^{h p}=Q_{n, t}^{s h}+Q_{n, t}^{d h w} \\
& \forall n, t: Q_{n, t}^{h p} \geq 0,
\end{aligned}
$$

where $P_{45}$ and $Q_{45}$ are heat pump power and heat sink at $45^{\circ} \mathrm{Cfrom}$ data sheet.

$$
\begin{array}{r}
T_{n, t}^{s t o}-\left(1-S_{n, t}^{s h}\right) * T_{n}^{\max } \leq w 1_{n, t}+w 2_{n, t}+w 3_{n, t} \\
T_{n, t}^{d h w}-\left(1-S_{n, t}^{d h w}\right) * T_{n}^{\max } \leq w 1_{n, t}+w 2_{n, t}+w 3_{n, t}
\end{array}
$$

The heat pump model is defined as a piecewise linear function of the sink and source temperatures. Positive weights $w$ are associated to each node. At each time step their sum is equal to the activity level of the heat pump.

\section{Typical days}

Calculating flexibility for a building with various devices for an entire year using any optimisation framework is a computationally challenging task. It becomes especially challenging when the complexity of the system models increases. To avoid the "curse of dimensionality", other researchers have made use of typical days that represent the entire year. Studies such as those performed by Fazlollahi et al. (2014) and Domínguez-Muñoz et al. (2011) highlight the interest of using typical days for system design optimisations. For the purpose of this study we have also used typical days to represent the year's weather, including solar irradiance and external temperature. Days are clustered by three in order to be used for initialization (day 1) and simulation (day 2) and prediction (day 3).

When characterizing the flexibility potential of residential buildings, one has to consider both space heating and domestic hot water at the same time, as most systems use a single heat pump to provide both services. Furthermore, in a typical building management system, DHW requirements will have priority over space heating, thus impacting the flexibility offered by the heat pump. When assessing flexibility potential, one has to take into account that DHW consumption can have significant effects both, on the heat pump and on the space heating; as in certain cases, not enough heat is supplied to the building. In order to take this issue into account, as well as the impact of the initial state of the storage tanks, the typical day simulations are run with four different samples of DHW consumption profiles per building. 


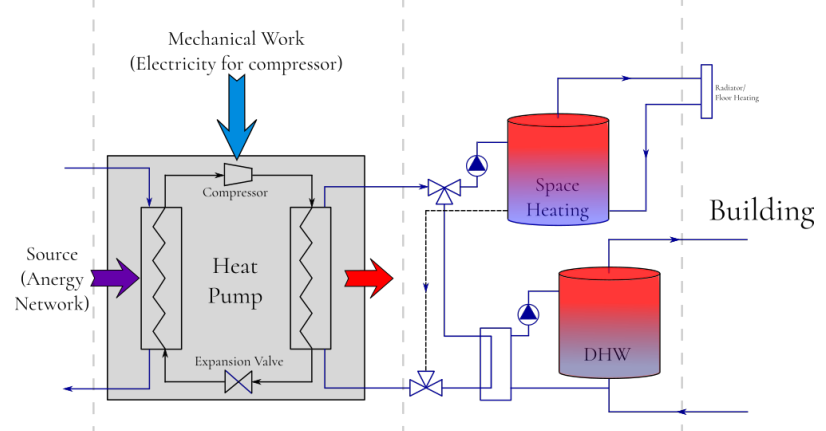

Figure 1: Schematic of the analyzed system at building level.

\section{Simulation framework}

Dynamic simulations of the cluster of buildings are carried out with MATLAB ${ }^{\circledR}$ SIMULINK ${ }^{\circledR}$ using a modified version of the Carnot toolbox (Wemhöner et al., 2000). In order to limit simulation time, buildings are reduced to a one zone model with four nodes: the external mass $T^{w_{\text {ext }}}$, the internal mass $T^{w_{\text {in }}}$, the floor $T^{f}$ (for floor heating) and the air inside the buildings $T^{i n}$. Heat is delivered to the building by radiators or floor heating. The heat distribution consists of three loops, which link the building with the buffer tank, the buffer tank with the heat pump and the heat pump with a heat source as displayed in Figure 1. This is a typical design for buildings supplied by heat pumps. The storage tank for Domestic Hot Water is linked to the heat pump through an external heat exchanger. The storage tanks are both modelled to account for thermal stratification. The dynamic behaviour of the heat pump is computed using piece-wise linearization of the source and sink thermal power and electrical power given by manufacturer data. The outlet temperatures of the condenser and the evaporator are calculated by two differential equations.

The MPC algorithm is implemented in python using the gurobipy library (Gurobi Optimization, 2018) to generate and solve the optimisation problem. Simulation values, DR parameters and control set-points are exchanged between SIMULINK ${ }^{\circledR}$ and python every 15 minutes in simulation time. The optimisation problem is updated at every call. Since the problem is formulated as a MILP, the optimisation is stopped when a solutions falls below $5 \%$ of the MIPgap or after 40 seconds in order to limit the computational time. If the problem is infeasible, the default control set-points are sent to the simulation controller to avoid failure of the run.

Each combination of typical day (Extreme winter, Winter, Mid-season, Summer) and DHW profiles (there are four) are simulated with one minute time steps. There are 24 runs for each combination, cor-

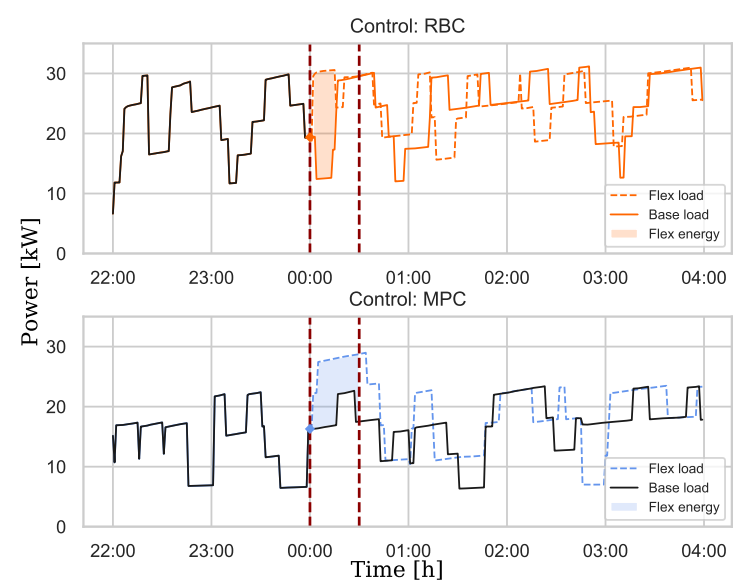

Figure 2: Example of the reaction of a cluster of buildings to a DR upward flexibility call in function of the control method.

responding to each hour of a day. The flexibility is evaluated at different hours of the day (Flexhour) and for different durations $d_{D R}$ (15, 30, 60 minutes). Each run has an initialization period of a day, and stops four hours after receiving a flexibility call at the beginning of the corresponding Flexhour.

For the sake of simplicity, we consider only one flexibility call per run, and only the maximum and minimum upward/downward flexibility is requested per call. The time of a flexibility request is assumed to be previously unknown to the system.

\section{Results}

Characterizing the flexibility of buildings over a year at high resolution (one minute time step) is not only computationally challenging but also difficult to summarise visually. Demand Response calls usually last for rather short periods of time, which motivates looking at hourly/daily timespans. Therefore, simulating typical days is a good way to analyse the flexibility of building clusters as they are shorter to simulate but still represent a pertinent summary of a year. In this section, we present results for two control strategies to provide flexibility. The flexibility is characterized by three different indicators and two different methods to compute the indicators are compared.

Figure 2 displays a DR call for providing upward flexibility for a typical mid-seasonal day sent to a small cluster of buildings. The DR call starts at midnight and lasts for 30 minutes (period between the two dashed vertical lines). The baseload and the power deviation of the two evaluated control strategies (RBC and MPC) are compared not only during the call but also during the following four hours. It can be seen in those subplots that the cluster with an MPC controller for cost minimization presents a baseload that is clearly lower than the one with a $\mathrm{RBC}$, which makes it difficult to compare the flexibility they offer. In this example, the total variations 


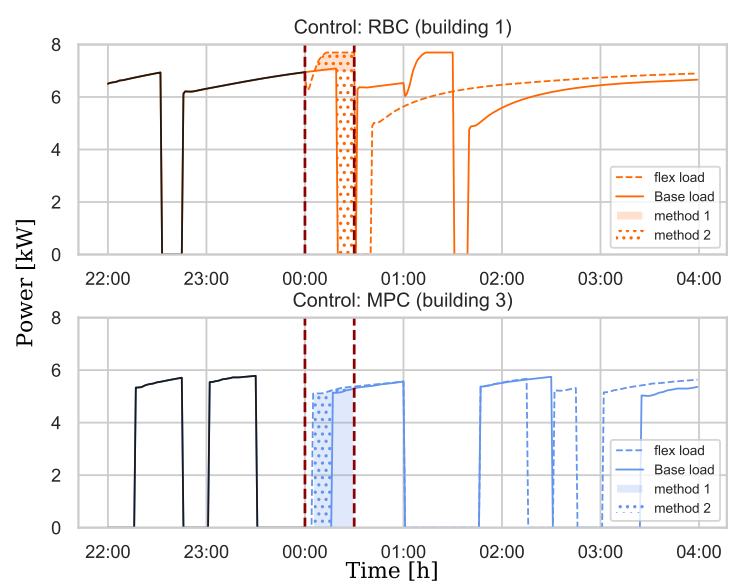

Figure 3: Reaction of a single building to a DR upward flexibility call.

of energy between the flex loads and the baseloads are similar in both cases, but, the respective patterns are different. In one case, the deviation is large but lasts for a short period of time. In the other case, it is smaller but persists for the call duration. The so-called rebound effect after the DR event is not always easily observable, in particular for such a small cluster of buildings. However, in the top subplot of Figure 2, the load resulting from the flex call at midnight seems to return to the baseload after less than four hours. In the case of DHW, we would need to look at longer periods, as charging/discharging cycles usually last longer than four hours.

Figure 3 displays the same upward flexibility call as presented in Figure 2, zoomed in to the building level. It illustrates two different cases of successful activation. In the top subplot, the heat pump is already activated and providing space heating when the DR call starts. After an initial decrease during the first six minutes, the power consumption increases by 10 $\%$ compared to the value at the beginning of the call. This behaviour corresponds to a switch from the heat mode to the DHW mode of the heat pump. When the call is received, the bottom temperature of the storage tank is lower than the current heat pump operation temperature, which explains the decrease in electrical power. One can observe that the DHW cycle was started one hour earlier. Computing flexibility with reference to the initial power (method 1) results in only a small energy increase. Looking at the baseload, the heat pump would have normally stopped during this period but was kept on by changing its set-points. Therefore, computing flexibility with reference to the baseload (method 2) gives a much bigger energy increase. The bottom subplot with MPC illustrates the opposite behaviour. At the start of the call the heat pump is off, but it switches on after 2 minutes. However, looking at the baseload, one can observe that the heat pump would have started on its own anyway. In this case, computing the flexible energy with

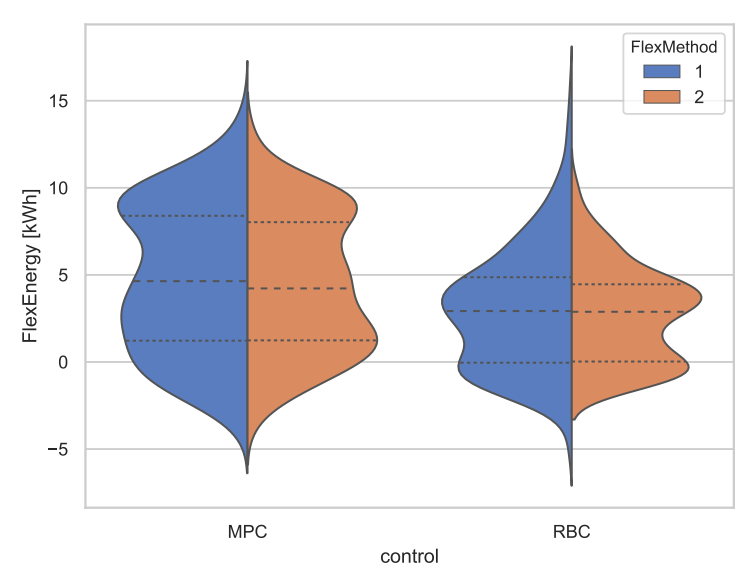

Figure 4: Aggregated flexible energy $\left(d_{D R}=30 \mathrm{~min}\right)$ compared by control type and method to compute indicators. In blue the difference relative to the current power level and in orange the difference relative to the baseload.

method 2 results in half the amount obtained with method 1.

In Figure 4, results are grouped by the control options MPC (left) and RBC (right) and by the flex methods used to compute the flexibility. The blue half corresponds to the difference relative to the current power level and the orange half to the difference relative to the baseload. When the flexible energy is aggregated and observed over all typical days, there are no major differences between the two ways of computing this indicator. However, the difference is slightly bigger when looking at the maximum power deviation. The violin plots have two peaks; one occurring around zero and the other at a higher value. This shape can be explained by the fact that the heat pumps considered have fixed speed compressors. They can be either on or off. The only power variation results from the increase in operating temperatures, which in the case of space heating, happens over several hours (longer that the call duration). A heat pump turning on, as a consequence of a DR call would therefore create a significant energy increase. This effect would probably vanish for bigger cluster sizes.

As one could expect, the MPC controller provides a bit more upward flexibility than the RBC controller for mainly two reasons: First, the system is operated at lower temperatures, which means the heat pumps have a higher chance to be turned off when a DR call is received. Second, the MPC controller finds the best choice of set-points to maximize the power deviation by predicting it over a longer horizon. However, it can also decide not to provide flexibility if it would mean violating comfort constraints. Negative values of upward FlexEnergy results from the heat pumps stopping during the DR call due to a cycle duration limit or too high operating temperatures.

Figure 5 shows, for each hour of each typical day, the 

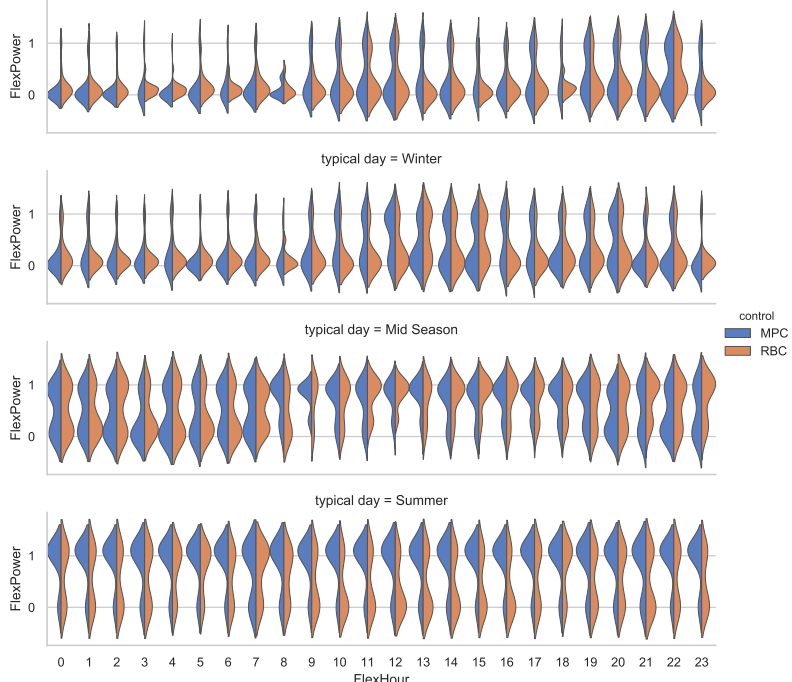

Figure 5: Maximum power deviation with the baseload as reference, per building, for each hour of the four typical days. The values are normalized by the operating power at $55^{\circ} \mathrm{C}$.

maximum power deviation with the base load as reference $\left(F_{2 t_{0}}^{U P}\right)$. This indicator is normalized by the power consumed in DHW mode with an operating temperature of $55^{\circ} \mathrm{C}$ which explains why its values can exceed one. Each violin plot is split to show the MPC (left, blue) and RBC (right, orange) distributions. The two top subplots correspond to extreme winter and winter days. One can observe that upward flexibility is almost zero throughout the hours of the day. This increases for the mid-season day, with around half the values closer to one than to zero. In summer, heat pumps only operate to provide DHW. The fact that they are turned off most of the time and that their operating temperatures are high, can explain why the highest power deviations are found in summer.

Hourly patterns can also be identified, especially during the Mid-season day. Between $8 \mathrm{AM}$ and $6 \mathrm{PM}$, power deviations are closer to their maximum values. The external temperature increases during the day, decreasing the departure temperature set-point of the heating system, which results in more "off" time of the heat pump. In reality, the building's heat distribution system can be operated with night setbacks. A night setback will lower the set-point temperature at night. The distribution of FlexPower would therefore be flipped, compared to the one in Figure 5. Night time would have the highest maximum deviation potential. One can observe the effect of the peaks of consumption in the morning, 7 to $8 \mathrm{AM}$, and in the early evening, around 6 PM, when DHW storage tanks become empty.

Figure 6 displays the aggregated flexible electrical energy in kWh of the five residential buildings considered for each FlexHour. The duration of the call considered here is $d_{D R}=30 \mathrm{~min}$. For this duration,

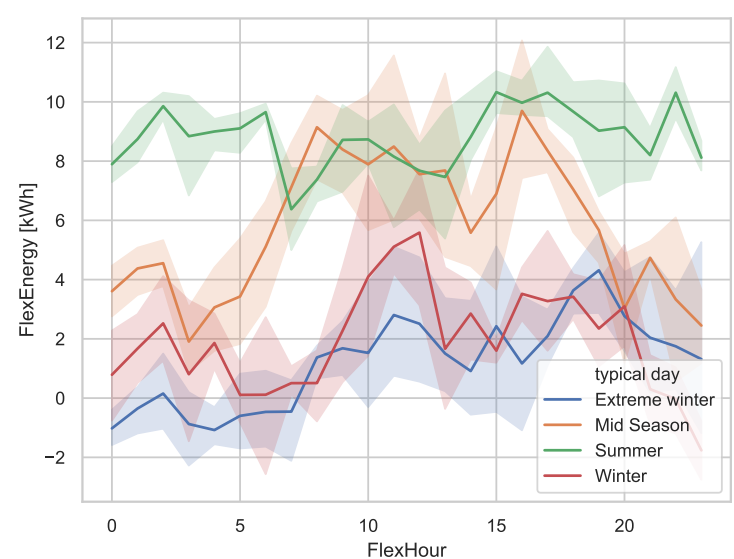

Figure 6: Variation of FlexEnergy potential with $M P C$, computed with method 2, for $d_{D R}=30 \mathrm{~min}$, over four typical days, with deviations.

the theoretical maximum flexible energy of the system considered is less than $20 \mathrm{kWh}$, assuming that all heat pumps turn on at the beginning of the call to provide DHW throughout the entire duration. As previously discussed in 5, the FlexEnergy is low for winter days but the deviations are wider. The bands represent the results obtained for different DHW profiles. In winter, DHW cycles have a significant impact on the overall system as they often interrupt heating cycles. In summer, upward flexibility results only from an increase of the DHW temperature set-point $T_{D H W_{\text {set }}}$. The thermal energy that can be stored in a DHW storage tank is an order of magnitude lower than the one that can be stored in the building mass. Once the storage tank is full, the heat pump cannot provide any flexibility for several hours. DHW charging cycles typically range from 15 minutes to maximum one hour if the storage is empty. This is why the value of the FlexEnergy would not increase much even for a longer duration $d_{D R}$. The same remark can be made for winter periods, where off times are usually very short. Depending on the method used to compute the flexibility, results could be very different. For Mid-season days, however, increasing $d_{D R}$ up to one hour per day would result in higher FlexEnergy upward potential because heat pumps are more often turned off during those days and more thermal energy can be stored in the mass of the building.

Table 1 shows the means and the extrema of the results presented in Figure 6. It shows that on average MPC provided at least $25 \%$ more FlexEnergy than RBC. For Mid-season, this increase goes up to more than $50 \%$.

\section{Conclusion}

This paper proposes a framework to assess the maximum energy flexibility potential for a cluster of buildings with a focus on complex thermoelectric devices such as water-to-water heat pumps. A key contri- 
Table 1: Mean, minimum and maximum of the FlexEnergy potential in $k W h$. This potential was computed with MPC and method 2 for $d_{D R}=30 \mathrm{~min}$, over four typical days. The percentages in brackets correspond to the increase of flexibility compared to the one obtained with $R B C$.

\begin{tabular}{|c|c|c|c|}
\hline \multirow{2}{*}{$\begin{array}{c}\text { Typical } \\
\text { day }\end{array}$} & \multicolumn{3}{|c|}{ FlexEnergy [kWh] } \\
\cline { 2 - 4 } & Mean & Min & Max \\
\hline Ext. winter & $1.2(30 \%)$ & $-3.1(-46 \%)$ & $7.4(12 \%)$ \\
Winter & $1.9(25 \%)$ & $-3.5(222 \%)$ & $9.0(20 \%)$ \\
Mid-season & $5.8(52 \%)$ & $0.3(77 \%)$ & $13.1(33 \%)$ \\
Summer & $8.8(37 \%)$ & $4.1(-56 \%)$ & $12.6(11 \%)$ \\
\hline
\end{tabular}

bution is the incorporation of the two control methods RBC and MPC in the characterization process of a small residential cluster. This addition is made computationally feasible by the use of typical days to simulate the year. Two different methods to compute the flexibility indicators, related to the reference chosen to account for deviation, are discussed. Simulations are carried out to demonstrate the value of the proposed formulations and metrics, especially in relation to maximum flexibility potential fluctuations over a day or between seasons. Our results show a high seasonal and lower hourly variation of flexibility with a maximum potential reach for Mid-season days. We also find that the flexibility offered by heat pumps could benefit from a control method such as MPC capable of predicting and optimizing the setpoint values. However, the robustness and scalability of the method for larger cluster sizes will have to be investigated alongside the evaluation of the MPC objective function.

\section{Acknowledgment}

This project has received funding from the European Union's Horizon 2020 research innovation programme under grant agreement No. 695965. The authors would also like to thank the partners of the Sim4Blocks project for their invaluable support and help without which this paper would not have been possible.

\section{References}

Domínguez-Muñoz, F., J. M. Cejudo-López, A. Carrillo-Andrés, and M. Gallardo-Salazar (2011). Selection of typical demand days for chp optimization. Energy and Buildings 43(11), 3036 -3043 .

Dhulst, R., W. Labeeuw, B. Beusen, S. Claessens, G. Deconinck, and K. Vanthournout (2015). Demand response flexibility and flexibility potential of residential smart appliances: Experiences from large pilot test in belgium. Applied Energy 155, 79 -90 .

Fazlollahi, S., S. L. Bungener, P. Mandel, G. Becker, and F. Maréchal (2014). Multi-objectives, multi- period optimization of district energy systems: I. selection of typical operating periods. Computers \&5 Chemical Engineering 65, 54-66.

Gurobi Optimization, L. (2018). Gurobi optimizer reference manual.

Junker, R. G., A. G. Azar, R. A. Lopes, K. B. Lindberg, G. Reynders, R. Relan, and H. Madsen (2018). Characterizing the energy flexibility of buildings and districts. Applied Energy 225, 175 $-182$.

Remund, J. and S. Kunz (1995). Meteonorm - a comprehensive meteorological database and planning tool for system design. In "Proceedings from the 13th European Photovoltaic Solar Energy Conference", pp. 733-735. Nice, 23 - 27 October 1995.

Reynders, G., R. A. Lopes, A. Marszal-Pomianowska, D. Aelenei, J. Martins, and D. Saelens (2018). Energy flexible buildings: An evaluation of definitions and quantification methodologies applied to thermal storage. Energy and Buildings 166, 372 - 390 .

Schütz, T., H. Harb, R. Streblow, and D. Müller (2015). Comparison of models for thermal energy storage units and heat pumps in mixed integer linear programming. In Proceedings from ECOS2015: The 28th international conference on Efficiency, Cost, Optimization, Simulation and Environmental Impact of Energy Systems. Pau, 29 June - 3rd July 2015.

Stinner, S., K. Huchtemann, and D. Müller (2016). Quantifying the operational flexibility of building energy systems with thermal energy storages. $A p$ plied Energy 181, 140 - 154.

Van der Hoeven, M. (2012). Urban energy policy design. In A. Nethersol (Ed), SUSTAINABLECITIES, Building cities for the future. Green Media Ltd. London (UK).

Wemhöner, C., B. Hafner, and K. Schwarzer (2000). Simulation of solar thermal systems with carnot blockset in the environment matlabß simulink@). In Proceedings from Eurosun2000: ISES Europe Solar Congress, pp. 1-6. Copenhagen, 19-22 June 2000.

Zhang, L., N. Good, and P. Mancarella (2019). Building-to-grid flexibility: Modelling and assessment metrics for residential demand response from heat pump aggregations. Applied Energy 233-234, $709-723$. 